\title{
SOME ABNORMALITIES OF SEX DEVELOPMENT DUE TO ERRORS OF THE SEX CHROMOSOMES
}

\author{
Paul E. Polani, M.D., F.R.C.P. \\ Padiatric Research Unit, Guy's Hospital Medical School, London, S.E.I
}

IT is estimated that chromosome abnormalities responsible for congenital errors of development may be present in about one half per cent. of the general population and it is thought that about three-quarters of these chromosome errors involve abnormalities of the sex chromosomes. This, then, means that about 4,000 babies are born every year and survive with developmental anomalies due to chromosome errors and that in about 3,000 in a year the sex chromosomes are implicated.

I have taken only representative clinical examples of each of the major classes of sexchromosome errors which are responsible for developmental mishaps.

\section{Nuclear Sexing}

It should be stated from the beginning that one of the most important developments which led to the discovery of these abnormalities of the sex chromosomes was the technique of nuclear sexing, developed by Murray L. Barr in 1949, and later applied to the study of certain errors of sex development. But nuclear sexing was not only important, historically, in pinpointing for chromosome studies certain abnormalities of sex development, but also is indispensable at the present time in arriving at a proper recognition of the various sex-chromosome abnormalities found. Nuclear sexing and chromosome analysis go hand-in-hand in establishing the errors of the sex chromosomes which are responsible for developmental anomalies of sex.

The nuclear-sexing technique depends on the recognition of a visible and stainable mass in certain nuclei of somatic cells. The reasons for the visibility of the sex-chromatin mass in some subjects arises out of the following considerations. It is assumed that, although the chromosomes in the interphase or resting nucleus are not stainable and visible as separate elements within the

A paper read to the Harveian Society of London on December 20, I 96 I. nucleus, nevertheless they are present as elongated filaments. These become coiled and progressively contracted preparatory to cell division and, consequently, become visible and stainable. However, although it is true that the greatest part of the chromosomes is completely unstainable at this stage, nevertheless some portions of the chromosomes are visible. They represent those minute masses called 'chromocentres' which are clearly seen inside the nuclei of many somatic cells. The most striking of these visible elements is that called 'sex chromatin', a little mass about $I \frac{1}{2}$ micra in diameter, planoconvex, often coffeebean shaped and visible at the nuclear membrane of cells of females but not of males. This mass is not seen in all the cells of females, but only in a majority of them. Usually it is absent from all the cells of males, but occasionally it can be seen in a very small proportion of them. The cells that are studied best are those with an open nucleus, like the cells of the oral mucosa, of the skin, of the liver, of the adrenal gland, etc. For practical purposes the skin, and the oral mucosa above all, are preferentially used. However, a sex difference can also be easily detected in the leucocytes of the peripheral blood. The sex-chromatin mass, which as we have seen is present in the open nuclei of somatic cells of females but not in those of normal males, represents one of the two $\mathrm{X}$ chromosomes when two are present in the diploid set. The actual origin of the sex-chromatin mass from one $\mathrm{X}$ chromosome and the implications of such a derivation are the subject of considerable thought, and even of some controversy at the present time. However, if, as may happen in abnormal circumstances, there are more than two $\mathrm{X}$ chromosomes in the set, then the number of sex-chromatin masses increases in proportion, there being always one less than the number of $\mathrm{X}$ chromosomes present. It should also be noted that the number of sexchromatin masses depends entirely and solely on the number of $\mathrm{X}$ chromosomes present and is not influenced by the presence of the Y chromosomes. One of the important points which have 
not yet been settled is whether the number of sex-chromatin masses is influenced by the number of autosomes present in the set. According to some authors, the number of sex-chromatin masses is related to the balance between the number of autosomes and that of $\mathrm{X}$ chromosomes, whereas others believe that the $\mathrm{X}$ chromosomes act in a manner independent of the number of autosomes which may be present in the set in abnormal circumstances. It follows from these considerations that, when one sex-chromatin mass is detected in the nuclei of oral-mucosa cells of a subject, the corresponding sex-chromosome constitution can be XX or XXY or XXYY for instance. When two masses are detected, there may be three $\mathrm{X}$ chromosomes in the set with a total number of $47 \mathrm{chromosomes}$ or there may be three $\mathrm{X}$ chromosomes and a $\mathrm{Y}$ chromosome in the set with a total number of 48 chromosomes. When three masses are found there may be four $\mathrm{X}$ chromosomes, or equally well four $\mathrm{X}$ chromosomes and a $\mathrm{Y}$ chromosome. When no sex-chromatin masses are found in the cells with an open nucleus like those of the oral mucosa, various interpretations are possible; the karyotype may be XY like that of a normal male with 46 chromosomes or one may be dealing with an XYY subject, or one may be dealing with a patient who has only 45 chromosomes and a single $\mathrm{X}$ chromosome in the set, a state symbolically referred to as XO.

\section{Abnormalities of the Sex Chromosomes}

Let us leave now the subject of nuclear sexing and turn to the actual abnormalities of the sex chromosomes, some of which have just been briefly sketched out. Broadly speaking, these abnormalities can be of number or of structure; or occasionally they may involve both numerical and structural alterations of the sex chromosomes. Let us now look at some of the more typical, clinical examples with which some of the anomalies of the sex chromosomes which are known to occur in man have been associated. The first of these conditions is ovarian dysgenesis which, after puberty, is characterized by the absence of secondary sex characters. The affected women have primary amenorrhœa and do not have breasts or sex-hair development. In addition, patients affected by this condition are almost always of short stature, not reaching a height of $5 \mathrm{ft}$. in adult life. Occasionally some of the patients are intellectually slow. Women with this condition are usually chromatin negative on nuclear sexing but a proportion (say, one in four in hospital series) is chromatin positive. Among the chromatin-negative patients the presence of other anomalies than those of sex development and stature is quite common. For instance, webbing of the neck is often found, a peculiar facies is quite often remarked upon and a variety of somatic, skeleta, tegumentary and visceral anomalies is see Among the latter coarctation of the aorta frequently diagnosed and renal anomalies of various types are detected. The chromosom analysis of chromatin-negative patients wi ovarian dysgenesis reveals them to have almost always 45 chromosomes and a single $\mathrm{X}$ chrom some. This type of sex-chromosome complemef is symbolically referred to as XO.

A proportion of patients with ovarian dyo genesis are chromatin positive, and patients with chromatin-positive ovarian dysgenesis as a group show only rarely congenital malformations, wit the exception, of course, of shortening of statur@ When the chromosomes of these patients a studied some of them are found to be chromosome mosaics. By this I mean that, on careful chromof some counting, one finds in tissue culture obtained from them a bi-modal distribution of chromosome counts, some of the cells having 46 chromosomes and some having 45. Careful analysis of the cells with 46 chromosomes reveals no abnor mality in the chromosome make-up, and the two $\mathrm{X}$ chromosomes are present as in the cells of normal females. On the other hand, when the chromosomes are analysed of the cells with $\vec{\Phi}$ chromosome count of 45 , only a single X chromo 2 some is found and the cells therefore resemble chromosomally the cells of the chromatin-negatove patients with ovarian dysgenesis: these cells have an XO sex-chromosome complement. Th\% finding of this double peak of chromosome count and this distinct type of chromosome make-up i two different populations of cells in culture suggests that the patient's tissues which have been studied are made up also of two population of cells. Hence the name "chromosome mosaicisms.

But not all patients with chromatin-positive ovarian dysgenesis are sex-chromosome mosaic sis. in fact, probably the majority is not. In these. patients by far the most common finding is the presence of 46 chromosomes in the cells of the tissues in culture. A normal X chromosome is present and in addition, another $\mathrm{X}$ chromosome present which, however, is structurally altered an abnormal. This chromosome is generally inter preted as an 'isochromosome' of the long arm of the $\mathrm{X}$ chromosome. These isochromosomes are thought to arise out of an error of the division of the centromere when the chromatids separato during the course of cell division. Normally the centromere splits longitudinally, so that the two replicas - the chromatids-which are identical to each other, move to opposite poles of the dividing cell. However, occasionally, for a variety of reasons, it may happen that the centromere, in 
stead of dividing longitudinally, divides transversely. If the chromosome originally was an asymmetrical chromosome with a long arm and a short arm, this transverse division of the centromere will result in the two short arm chromatids and the two long arm chromatids separating from each other and migrating to opposite poles of the dividing cell. In this way, two perfectly symmetrical chromosomes are formed to which the name is given of 'isochromosomes'-one of the isochromosomes would be a symmetrical isochromosome for the short arm of the chromosome and the other one would be symmetrically made up of the long arm of the original chromosome. So far, in chromatin-positive ovarian dysgenesis, only subjects who have a symmetrical isochromosome for the long arm of it have been described but a patient has been seen who may possibly carry an isochromosome for the short arm of the $\mathrm{X}$ chromosome. This patient had 46 chromosomes in all, she had ovarian maldevelopment and was chromatin positive but did not show any somatic malformation nor the striking feature of ovarian dysgenesis, i.e. the short stature. She had, in fact, primary amenorrhœa and a normal stature.

A word should be said about the frequency with which the XO condition is found in the general population. This appears to be of the order of one in 3,000 females, and the prevalence of mosaics and isochromosome subjects with chromatinpositive ovarian dysgenesis may be of the order of one in 3,000 subjects, so that the total prevalence of ovarian dysgenesis, either chromatin positive or negative, is of the order of one in 1,500 females.

Klinefelter's syndrome is a condition in males who have small testes after puberty and azoöspermia. These males are sterile and quite often have gynæcomastia. The majority of patients with Klinefelter's syndrome are chromatin positive on nuclear sexing. It should be noted also that many patients with Klinefelter's syndrome are intellectually subnormal. When the chromosomes are studied of chromatin-positive patients with Klinefelter's syndrome it is usually found that they have 47 chromosomes and two $\mathrm{X}$ chromosomes like a normal female and a $\mathrm{Y}$ chromosome like a normal male. This XXY sex-chromosome constitution is, in fact, the explanation of their condition and of the nuclear sexing findings. In addition to XXY subjects with 47 chromosomes some patients are found with Klinefelter's syndrome in whom there is evidence of sex-chromosome mosaicism. Some of their cells have 46 chromosomes and others 47 . Thiose with 46 chromosomes generally are either $\mathrm{XY}$ or $\mathrm{XX}$, whereas those with 47 have an XXY sex-chromosome complement.

The total prevalence of the chromatin-positive type of Klinefelter's syndrome in the general population is of the order of one in 300 or 400 males. Of these chromatin-positive patients, a proportion is of non-mosaic patients and the rest are sex-chromosome mosaics as I have indicated.

The other major abnormality of the sex chromosomes is the so-called 'triplo-X syndrome'. This is a syndrome of mental deficiency generally, without any apparent interference with sex development. Females affected by it are chromatin-positive but their oral mucosal smear shows two sex-chromatin masses in a proportion of cells. Study of the chromosomes of these females has revealed that they have 47 chromosomes with three $\mathrm{X}$ chromosomes.

The prevalence of the condition in the general population is of the order of about one in 700 females.

In addition to these abnormalities which involve the sex chromosomes there are recorded a few instances of abnormalities involving the autosomes as well as the sex chromosomes. Mongolism has been found in association with the syndrome of Klinefelter, both clinically and chromosomally. A study of their chromosomes reveals a chromosome number of 48 , with three chromosomes No. $2 I$ in the set instead of the normal two, and an XXY sex-chromosome complement. Needless to say, such patients are, unlike ordinary male mongols, chromatin positive on nuclear sexing. 